\title{
Sampling and Descriptive Statistics
}

\author{
Berlin Chen \\ Department of Computer Science \& Information Engineering \\ National Taiwan Normal University

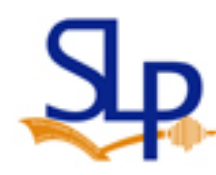

Reference:

1. W. Navidi. Statistics for Engineering and Scientists. Chapter 1 \& Teaching Material 


\section{Sampling (1/2)}

- Definition: A population is the entire collection of objects or outcomes about which information is sought

- Definition: A sample is a subset of a population, containing the objects or outcomes that are actually observed

- E.g., the study of the heights of NTNU students

- Choose the 100 students from the rosters of football or basketball teams (appropriate?)

- Choose the 100 students living a certain dorm or enrolled in the statistics course (appropriate?) 


\section{Sampling (2/2)}

- Definition: A simple random sample (SRS) of size $n$ is a sample chosen by a method in which each collection of $n$ population items is equally likely to comprise the sample, just as in the lottery

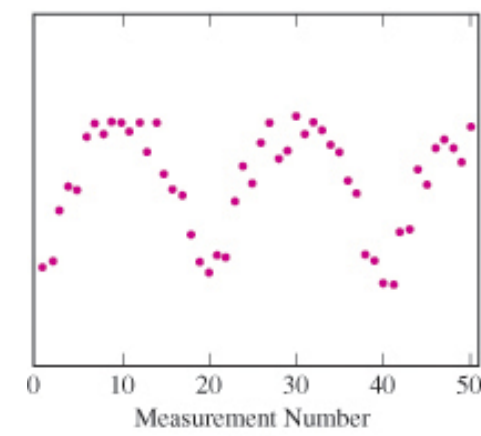

(a)

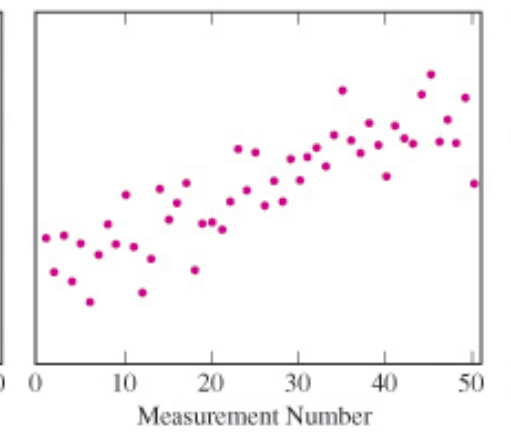

(b)

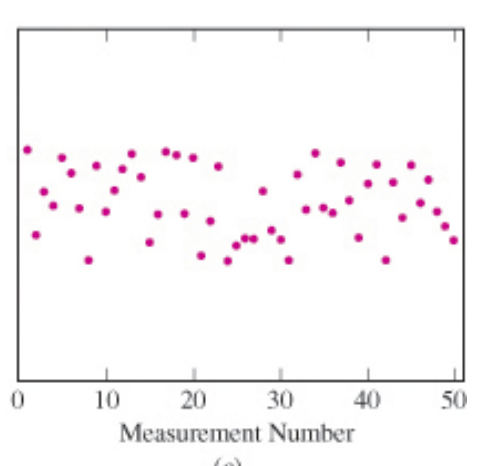

(c)

- Definition: A sample of convenience is a sample that is not drawn by a well-defined random method

- Things to consider with convenience samples:

- Differ systematically in some way from the population

- Only use when it is not feasible to draw a random sample 


\section{More on SRS (1/3)}

- Definition: A conceptual population consists of all the values that might possibly have been observed

- It is in contrast to "tangible (可觸之的) population"

- E.g., a geologist weighs a rock several times on a sensitive scale. Each time, the scale gives a slightly different reading

- Here the population is conceptual. It consists of all the readings that the scale could in principle produce 


\section{More on SRS (2/3)}

- A SRS is not guaranteed to reflect the population perfectly

- SRS's always differ in some ways from each other, occasionally a sample is substantially different from the population

- Two different samples from the same population will vary from each other as well

- This phenomenon is known as sampling variation 


\section{More on SRS (3/3)}

- The items in a sample are independent if knowing the values of some of the items does not help to predict the values of the others

- (A rule of Thumb) Items in a simple random sample may be treated as independent in most cases encountered in practice

- The exception occurs when the population is finite and the sample comprises a substantial fraction (more than $5 \%$ ) of the population

- However, it is possible to make a population behave as though it were infinite large, by replacing each item after it is sampled

- Sampling With Replacement 


\section{Other Sampling Methods}

- Weighting Sampling

- Some items are given a greater chance of being selected than others

- E.g., a lottery in which some people have more tickets than others

- Stratified Sampling

- The population is divided up into subpopulations, called strata

- A simple random sample is drawn from each stratum

- Cluster Sampling

- Items are drawn from the population in groups or clusters

- E.g., the U.S. government agencies use cluster sampling to sample the U.S. population to measure sociological factors such as income and unemployment 


\section{Types of Experiments}

- One-Sample Experiment

- There is only one population of interest

- A single sample is drawn from it

- Multi-Sample Experiment

- There are two or more populations of interest

- A simple is drawn from each population

- The usual purpose of multi-sample experiments is to make comparisons among populations 


\section{Types of Data}

- Numerical or quantitative if a numerical quantity is assigned to each item in the sample

- Height

- Weight

- Age

- Categorical or qualitative if the sample items are placed into categories

- Gender

- Hair color

- Blood type

\begin{tabular}{ccc}
\hline Specimen & $\begin{array}{l}\text { Torque } \\
(\mathbf{k N} \cdot \mathbf{m})\end{array}$ & $\begin{array}{l}\text { Failure } \\
\text { Location }\end{array}$ \\
\hline 1 & 165 & Weld \\
2 & 237 & Beam \\
3 & 222 & Beam \\
4 & 255 & Beam \\
5 & 194 & Weld \\
\hline
\end{tabular}




\section{Summary Statistics (1/4)}

- The summary statistics are sometimes called descriptive statistics because they describe the data

- Definition: Sample Mean

- Let $X_{1}, \ldots, X_{n}$ be a sample. The sample mean is

$$
\bar{X}=\frac{1}{n} \sum_{i=1}^{n} X_{i}
$$

- It's customary to use a letter with a bar over it to denote a sample mean

- Definition: Sample Variance

- Let $X_{1}, \ldots, X_{n}$ be a sample. The sample variance is

$$
s^{2}=\frac{1}{n-1} \sum_{i=1}^{n}\left(X_{i}-\bar{X}\right)^{2}
$$

Which is equivalent to $s^{2}=\frac{1}{n-1}\left(\sum_{i=1}^{n} X_{i}^{2}-n \bar{X}^{2}\right)$ 


\section{Summary Statistics (2/4)}

- Actually, we are interest in

- Population mean

- Population deviation: Measuring the spread of the population

- Population deviation: The variations of population items around the population mean

- Practically, because population mean is unknown, we use sample mean to replace it

- Mathematically, the deviations around the sample mean tend to be a bit smaller than the deviations around the population mean

- So when calculating sample variance, the quantity divided by $n-1$ rather than $n$ provides the right correction

- To be proved later on! 


\section{Summary Statistics (3/4)}

- Definition: Sample Standard Deviation

- Let $X_{1}, \ldots, X_{n}$ be a sample. The sample deviation is

$$
s=\sqrt{\frac{1}{n-1} \sum_{i=1}^{n}\left(X_{i}-\bar{X}\right)^{2}}
$$

Which is equivalent to

$$
s=\sqrt{\frac{1}{n-1}\left(\sum_{i=1}^{n} X_{i}^{2}-n \bar{X}^{2}\right)}
$$

- The sample deviation measure the degree of spread in a sample 


\section{Summary Statistics (3/4)}

- If $X_{1}, \ldots, X_{n}$ is a sample, and $Y_{i}=a+b X_{i}$, where $a$ and $b$ are constants, then $\bar{Y}=a+b \bar{X}$

- If $X_{1}, \ldots, X_{n}$ is a sample, and $Y_{i}=a+b X_{i}$, where $a$ and $b$ are constants, then $s_{y}^{2}=b^{2} s_{x}^{2}$ and $s_{y}=|b| s_{x}$

- Definition: Outliers

- Sometimes a sample may contain a few points that are much larger or smaller than the rest

- Such points are called outliers

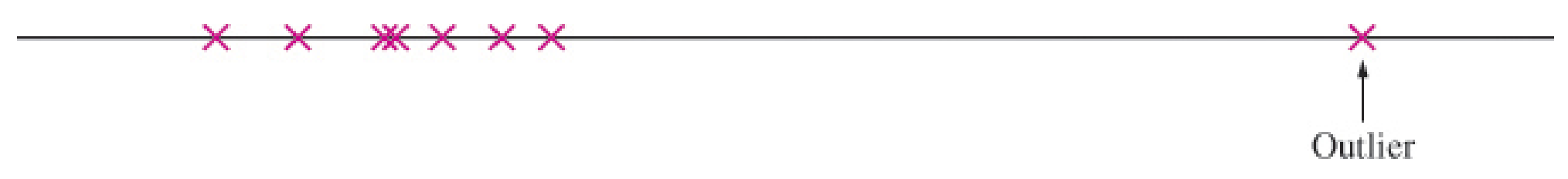




\section{More on Summary Statistics (1/2)}

- Definition: The median is another measure of center of a sample $X_{1}, \ldots, X_{n}$, like the mean

- To compute the median items in the sample have to be ordered by their values

- If $n$ is odd, the sample median is the number in position $(n+1) / 2$

- If $n$ is even, the sample median is the average of the numbers in positions $n / 2$ and $(n / 2)+1$

- The median is an important measure of center for samples containing outliers

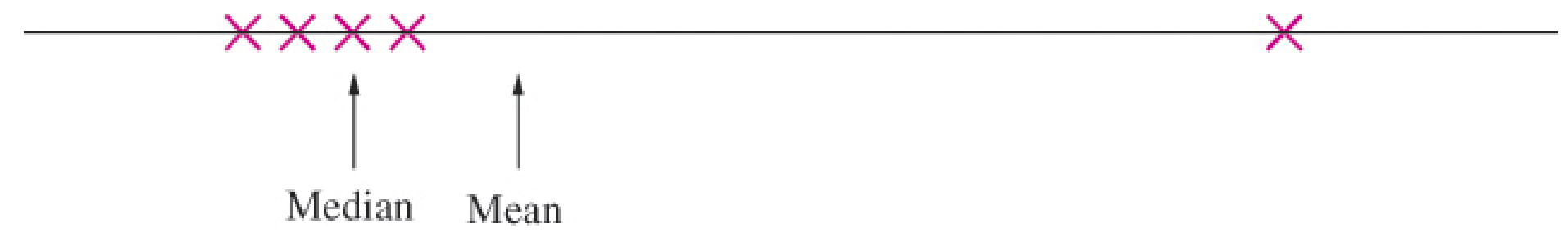




\section{More on Summary Statistics (2/2)}

- Definition: The trimmed mean of one-dimensional data is computed by

- First, arranging the sample values in order

- Then, trimming an equal number of them from each end, say, $p \%$

- Finally, computing the sample mean of those remaining 


\section{Quartiles}

- Definition: the quartiles of a sample $X_{1}, \ldots, X_{n}$ divides it as nearly as possible into quarters. The sample values have to be ordered from the smallest to the largest

- To find the first quartile, compute the value $0.25(n+1)$

- The second quartile found by computing the value $0.5(n+1)$

- The third quartile found by computing the value $0.75(n+1)$

- Example 1.14: Find the first and third quartiles of the data in Example 1.12

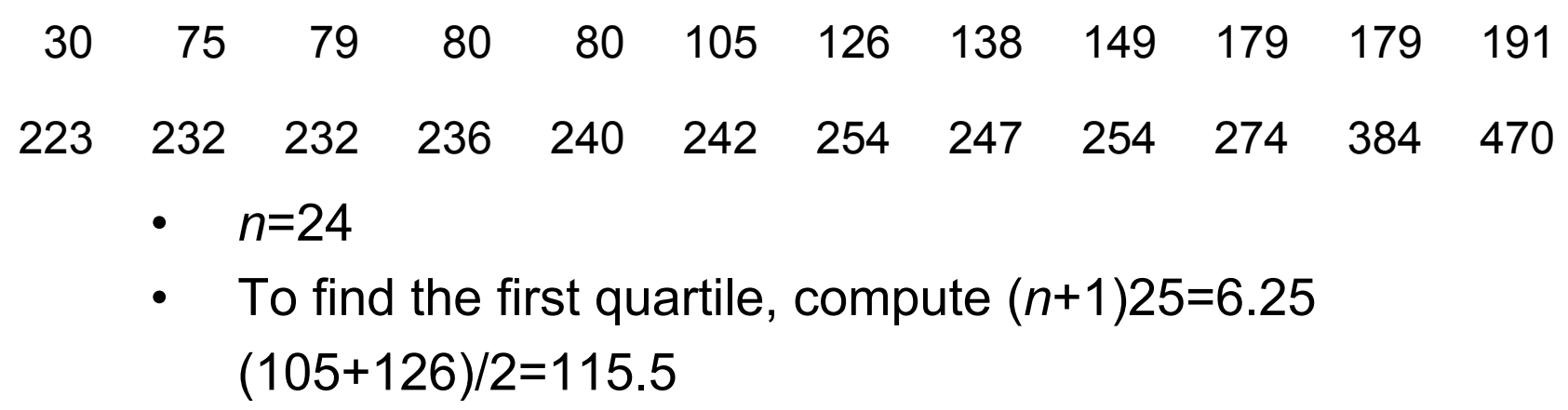

- $\quad$ To find the first quartile, compute $(n+1) 25=6.25$ $(105+126) / 2=115.5$

- To find the third quartile, compute $(n+1) 75=18.75$ $(242+245) / 2=243.5$ 


\section{Percentiles}

- Definition: The pth percentile of a sample $X_{1}, \ldots, X_{n}$, for a number between 0 and 100, divide the sample so that as nearly as possible $p \%$ of the sample values are less than the pth percentile. To find:

- Order the sample values from smallest to largest

- Then compute the quantity $(p / 100)(n+1)$, where $\mathrm{n}$ is the sample size

- If this quantity is an integer, the sample value in this position is the pth percentile. Otherwise, average the two sample values on either side

- Note, the first quartile is the 25th percentile, the median is the 50th percentile, and the third quartile is the 75th percentile 


\section{Summary Statistics for Categorical Data}

- For categorical data, each sample item is assigned a category rather than a numerical value

- Numerical Summaries for Categorical Data

- Definition: (Relative) Frequencies

- The frequency of a given category is simply the number of sample items falling in that category

- Definition: Sample Proportions

- The sample proportion is the frequency divided by the sample size 


\section{Sample Statistics and Population Parameters (1/2)}

- A numerical summary of a sample is called a statistic

- A numerical summary of a population is called a parameter

- If a population is finite, the methods used for calculating the numerical summaries of a sample can be applied for calculating the numerical summaries pf the population

- Exceptions are the variance and standard deviation

- However, sample statistics are often used to estimate parameters

- In practice, the entire population is never observed, so the population parameters cannot be calculated directly 


\section{Sample Statistics and Population Parameters (2/2)}

- A Schematic Depiction

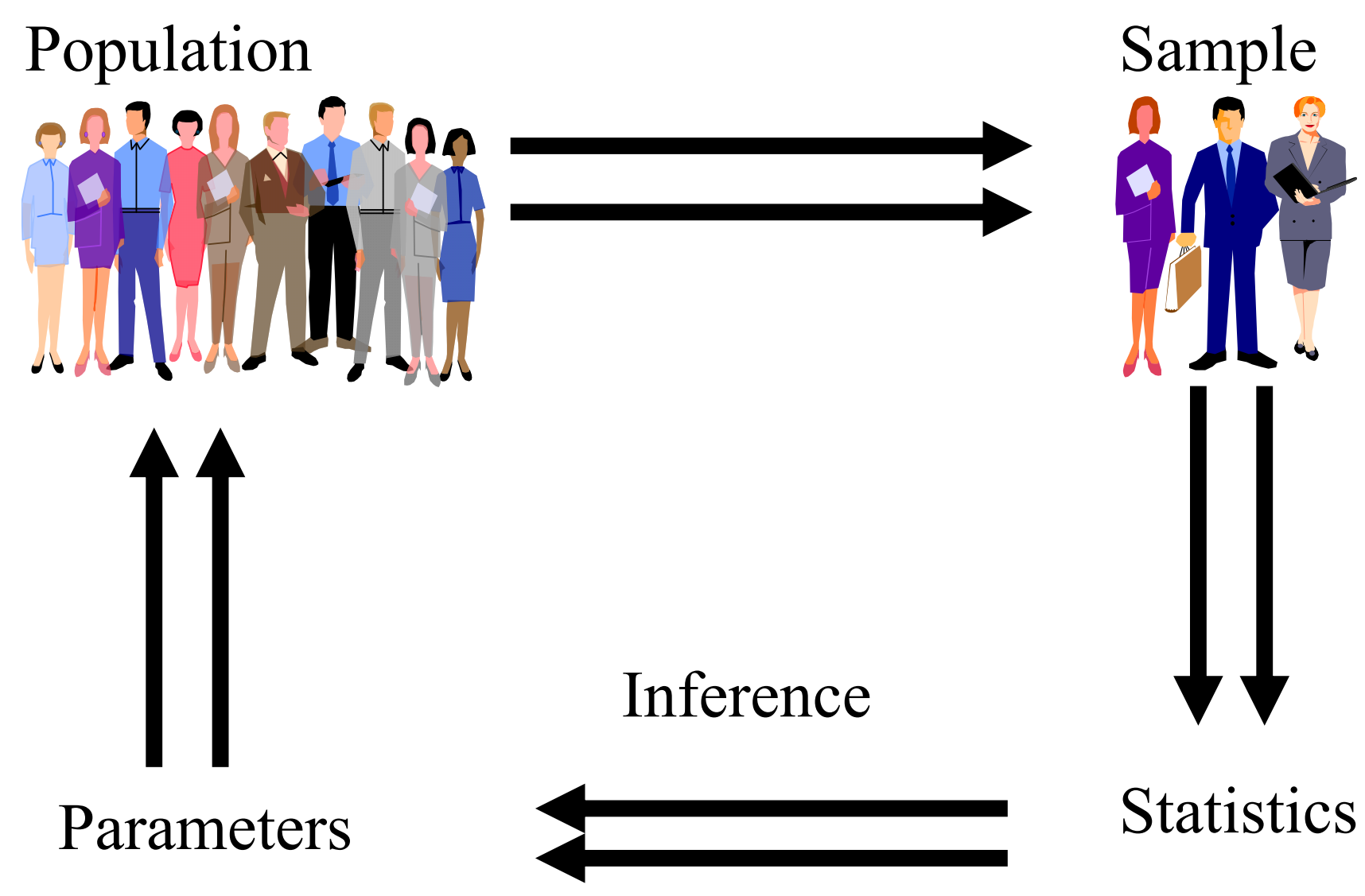




\section{Graphical Summaries}

- Recall that the mean, median and standard deviation, etc., are numerical summaries of a sample of of a population

- On the other hand, the graphical summaries are used as will to help visualize a list of numbers (or the sample items). Methods to be discussed include:

- Stem and leaf plot

- Dotplot

- Histogram (more commonly used)

- Boxplot (more commonly used)

- Scatterplot 


\section{Stem-and-leaf Plot (1/3)}

- A simple way to summarize a data set

- Each item in the sample is divided into two parts

- a stem, consisting of the leftmost one or two digits

- and the leaf, which consists of the next significant digit

- The stem-and-leaf plot is a compact way to represent the data

- It also gives us some indication of the shape of our data 


\section{Stem-and-leaf Plot (2/3)}

- Example: Duration of dormant (靜止) periods of the geyser (間歇泉) Old Faithful in Minutes

TABLE 1.3 Durations (in minutes) of dormant periods of the geyser Old Faithful

\begin{tabular}{|c|c|c|c|c|c|c|c|c|c|}
\hline 42 & 45 & 49 & 50 & 51 & 51 & 51 & 51 & 53 & 53 \\
\hline 55 & 55 & 56 & 56 & 57 & 58 & 60 & 66 & 67 & 67 \\
\hline 68 & 69 & 70 & 71 & 72 & 73 & 73 & 74 & 75 & 75 \\
\hline 75 & 75 & 76 & 76 & 76 & 76 & 76 & 79 & 79 & 80 \\
\hline 80 & 80 & 80 & 81 & 82 & 82 & 82 & 83 & 83 & 84 \\
\hline \multirow[t]{8}{*}{84} & 84 & 85 & 86 & 86 & 86 & 88 & 90 & 91 & 93 \\
\hline & & \multicolumn{2}{|c|}{ Stem } & \multicolumn{4}{|c|}{ Leaf } & & \\
\hline & & & 4 & \multicolumn{4}{|c|}{259} & & \\
\hline & & & 5 & \multicolumn{4}{|c|}{0111133556678} & & \\
\hline & & & 6 & \multicolumn{4}{|c|}{067789} & & \\
\hline & & & 7 & \multicolumn{4}{|c|}{01233455556666699} & & \\
\hline & & & 8 & \multicolumn{4}{|c|}{000012223344456668} & & \\
\hline & & & 9 & \multicolumn{4}{|c|}{013} & & \\
\hline
\end{tabular}

FIGURE 1.5 Stem-and-leaf plot for the geyser data in Table 1.3.

- Let's look at the first line of the stem-and-leaf plot. This represents measurements of 42,45 , and 49 minutes

- A good feature of these plots is that they display all the sample values. One can reconstruct the data in its entirety from a stemand-leaf plot 


\section{Stem-and-leaf Plot (3/3)}

- Another Example: Particulate matter (PM) emissions for 62 vehicles driven at high altitude

TABLE 1.2 Particulate matter (PM) emissions (in g/gal) for 62 vehicles driven at high altitude

\begin{tabular}{rrrrrrrrrrrrr}
\hline 7.59 & 6.28 & 6.07 & 5.23 & 5.54 & 3.46 & 2.44 & 3.01 & 13.63 & 13.02 & 23.38 & 9.24 & 3.22 \\
2.06 & 4.04 & 17.11 & 12.26 & 19.91 & 8.50 & 7.81 & 7.18 & 6.95 & 18.64 & 7.10 & 6.04 & 5.66 \\
8.86 & 4.40 & 3.57 & 4.35 & 3.84 & 2.37 & 3.81 & 5.32 & 5.84 & 2.89 & 4.68 & 1.85 & 9.14 \\
8.67 & 9.52 & 2.68 & 10.14 & 9.20 & 7.31 & 2.09 & 6.32 & 6.53 & 6.32 & 2.01 & 5.91 & 5.60 \\
5.61 & 1.50 & 6.46 & 5.29 & 5.64 & 2.07 & 1.11 & 3.32 & 1.83 & 7.56 & & &
\end{tabular}

Contain the a count of number of

Stem-and-leaf of HiAltitude $\quad N=62$

items at or above this line

Leaf Unit $=1.0$

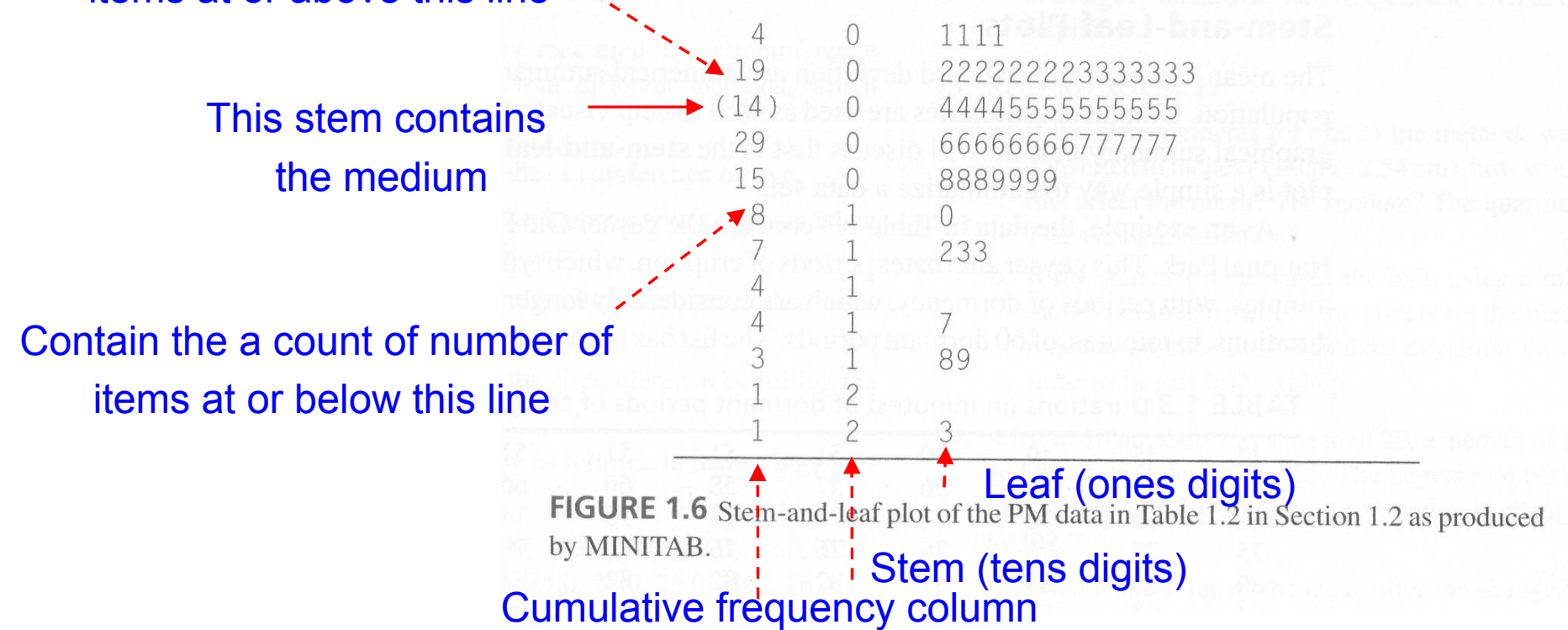

Cumulative fréquency column 


\section{Dotplot}

- A dotplot is a graph that can be used to give a rough impression of the shape of a sample

- It is useful when the sample size is not too large and when the sample contains some repeated values

- Good method, along with the stem-and-leaf plot to informally examine a sample

- Not generally used in formal presentations

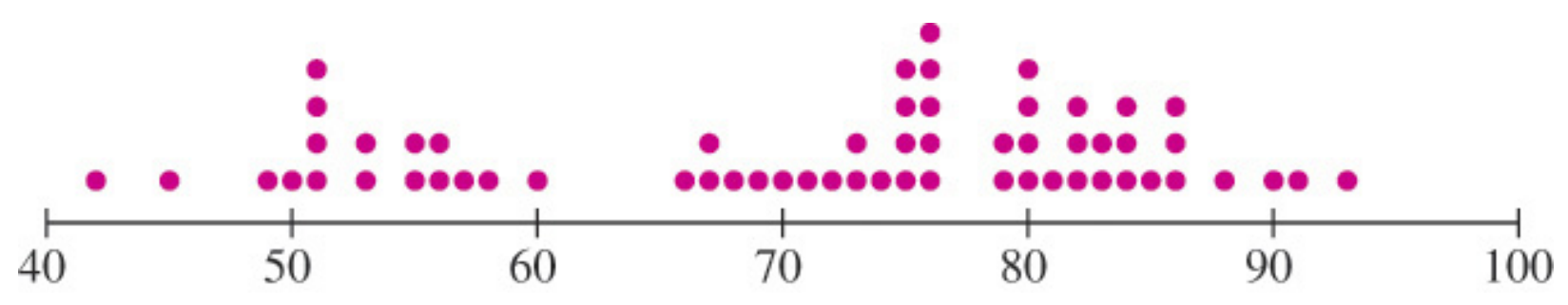

Figure 1.7 Dotplot of the geyser data in Table 1.3 


\section{Histogram (1/3)}

- A graph gives an idea of the shape of a sample

- Indicate regions where samples are concentrated or sparse

- To have a histogram of a sample

- The first step is to construct a frequency table

- Choose boundary points for the class intervals

- Compute the frequencies and relative frequencies for each class

- Compute the density for each class, according to the formula

Density = relative frequency/class width

- Density can be thought of as the relative frequency per unit 


\section{Histogram (2/3)}

TABLE 1.4 Frequency table for PM emissions of 62 vehicles driven at high altitude

\begin{tabular}{lccc}
\hline $\begin{array}{l}\text { Class } \\
\text { Interval (g/gal) }\end{array}$ & Frequency & $\begin{array}{l}\text { Relative } \\
\text { Frequency }\end{array}$ & Density \\
\hline $1-<3$ & 12 & 0.194 & 0.0970 \\
$3-<5$ & 11 & 0.177 & 0.0885 \\
$5-<7$ & 18 & 0.290 & 0.1450 \\
$7-<9$ & 9 & 0.145 & 0.0725 \\
$9-<11$ & 5 & 0.081 & 0.0405 \\
$11-<15$ & 3 & 0.048 & 0.0120 \\
$15-<25$ & 4 & 0.065 & 0.0065 \\
\hline
\end{tabular}

Table 1.4

- The second step is to draw a histogram for the table

- Draw a rectangle for each class, whose height is equal to the density

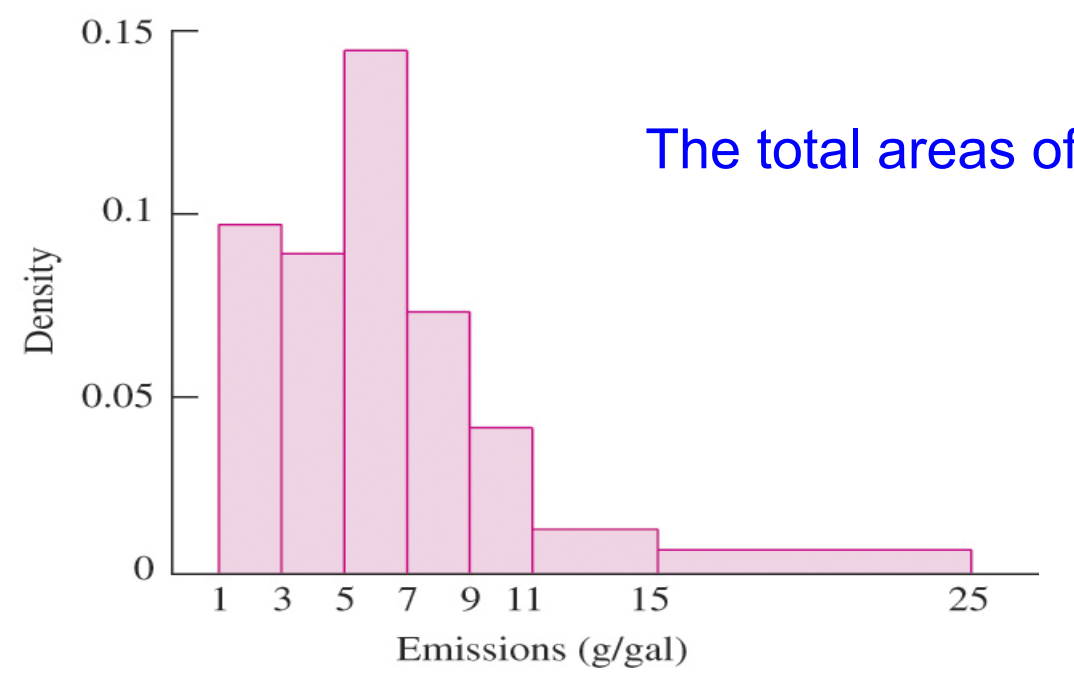

Figure 1.8 


\section{Histogram (3/3)}

- A common rule of thumb for constructing the histogram of a sample

- It is good to have more intervals rather than fewer

- But it also to good to have large numbers of sample points in the intervals

- Striking the proper balance between the above is a matter of judgment and of trial and error 


\section{Histogram with Equal Class Widths}

- Default setting of most software package

- Example: an histogram with equal class widths for Table 1.4

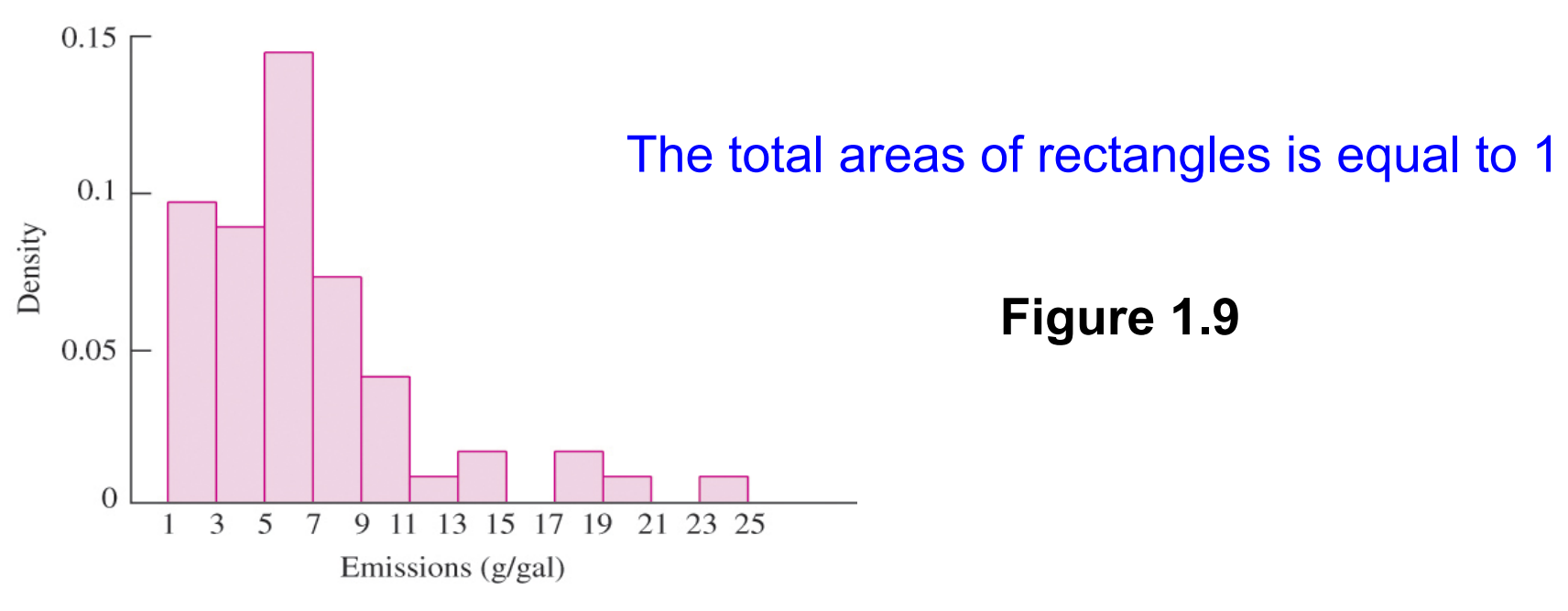

- Devoted to too many (more than half) of the class intervals to few (7) data points

- Compared to Figure 1.9, Figure 1.8 presents a smoother appearance and better enables the eye to appreciate the structure of the data set as a whole 


\section{Histogram, Sample Mean and Sample Variance (1/2)}

- Definition: The center of mass of the histogram is

$$
\sum_{i} \text { CenterOfClassInterval }_{i} \times \text { DensityOfClassInterval }_{i}
$$

- An approximation to the sample mean

- E.g., the center of mass of the histogram in Figure 1.8 is

$$
2 \times 0.194+4 \times 0.177+\cdots+20 \times 0.065=6.730
$$

- While the sample mean is 6.596

- The narrower the rectangles (intervals) are, the closer the approximation is 


\section{Histogram, Sample Mean and Sample Variance (2/2)}

- Definition: The moment of inertia (力矩慣量) for the entire histogram is

$$
\begin{gathered}
\sum_{i}\left(\text { CenterOfClassInterval }_{i}-{\text { CenterOfMassOfHistogram })^{2}}\right. \\
\times \text { DensityOfClassInterval }_{i}
\end{gathered}
$$

- An approximation to the sample variance

- E.g., the moment of inertia for the entire histogram in Figure 1.8 is

$$
(2-6.730)^{2} \times 0.194+(4-6.730)^{2} \times 0.177+\cdots+(20-6.730)^{2} \times 0.065=20.25
$$

- While the sample mean is 20.42

- The narrower the rectangles (intervals) are, the closer the approximation is 


\section{Symmetry and Skewness (1/2)}

- A histogram is perfectly symmetric if its right half is a mirror image of its left half

- E.g., heights of random men

- Histograms that are not symmetric are referred to as skewed

- A histogram with a long right-hand tail is said to be skewed to the right, or positively skewed

- E.g., incomes are right skewed (?)

- A histogram with a long left-hand tail is said to be skewed to the left, or negatively skewed

- Grades on an easy test are left skewed (?) 


\section{Symmetry and Skewness (2/2)}

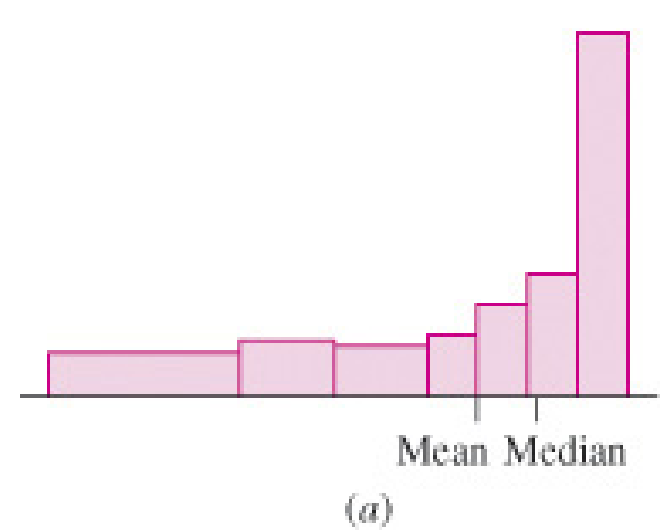

skewed to the left

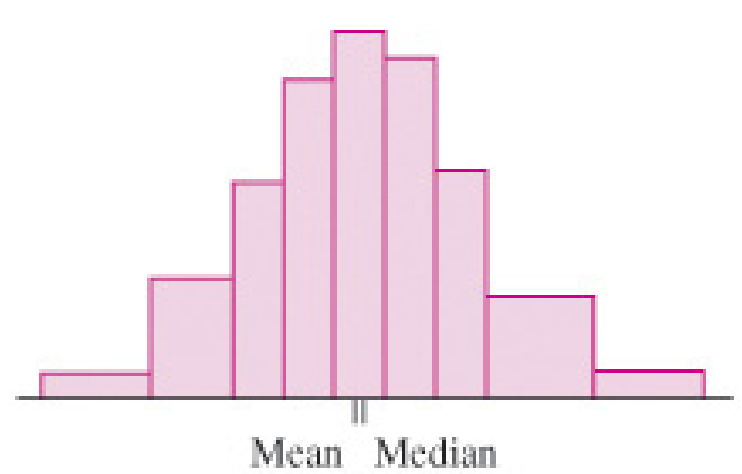

(b)

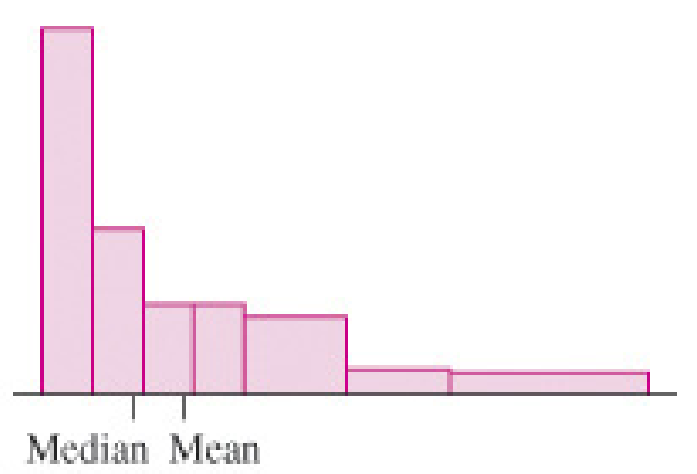

(c)

skewed to the right

- There is also another term called "kurtosis" that is also widely used for descriptive statistics

- Kurtosis is the degree of peakedness (or contrarily, flatness) of the distribution of a population

- For more on Skewness and Kurtosis, refer to: 


\section{Unimodal and Bimodal Histograms (1/2)}

- Definition: Mode

- Refer to the most frequently occurring value in a sample

- Or refer to a peak or local maximum for a histogram or other curves

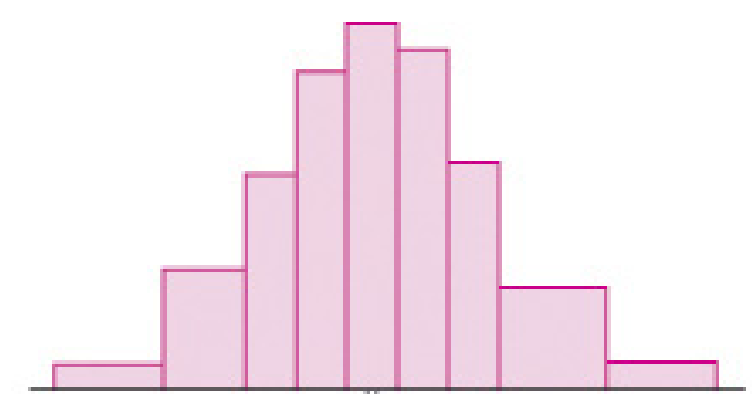

A unimodal histogram

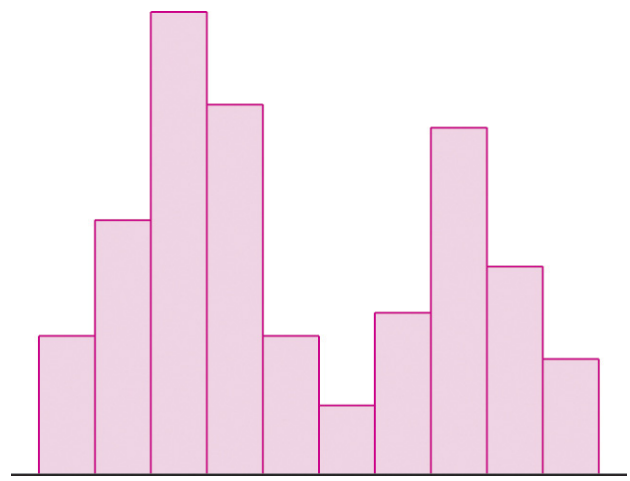

A bimodal histogram

- A bimodal histogram, in some cases, indicates that the sample can be divides into two subsamples that differ from each other in some scientifically important way 


\section{Unimodal and Bimodal Histograms (2/2)}

- Example: Durations of dormant periods (in minutes) and the previous eruptions of the geyser Old Faithful

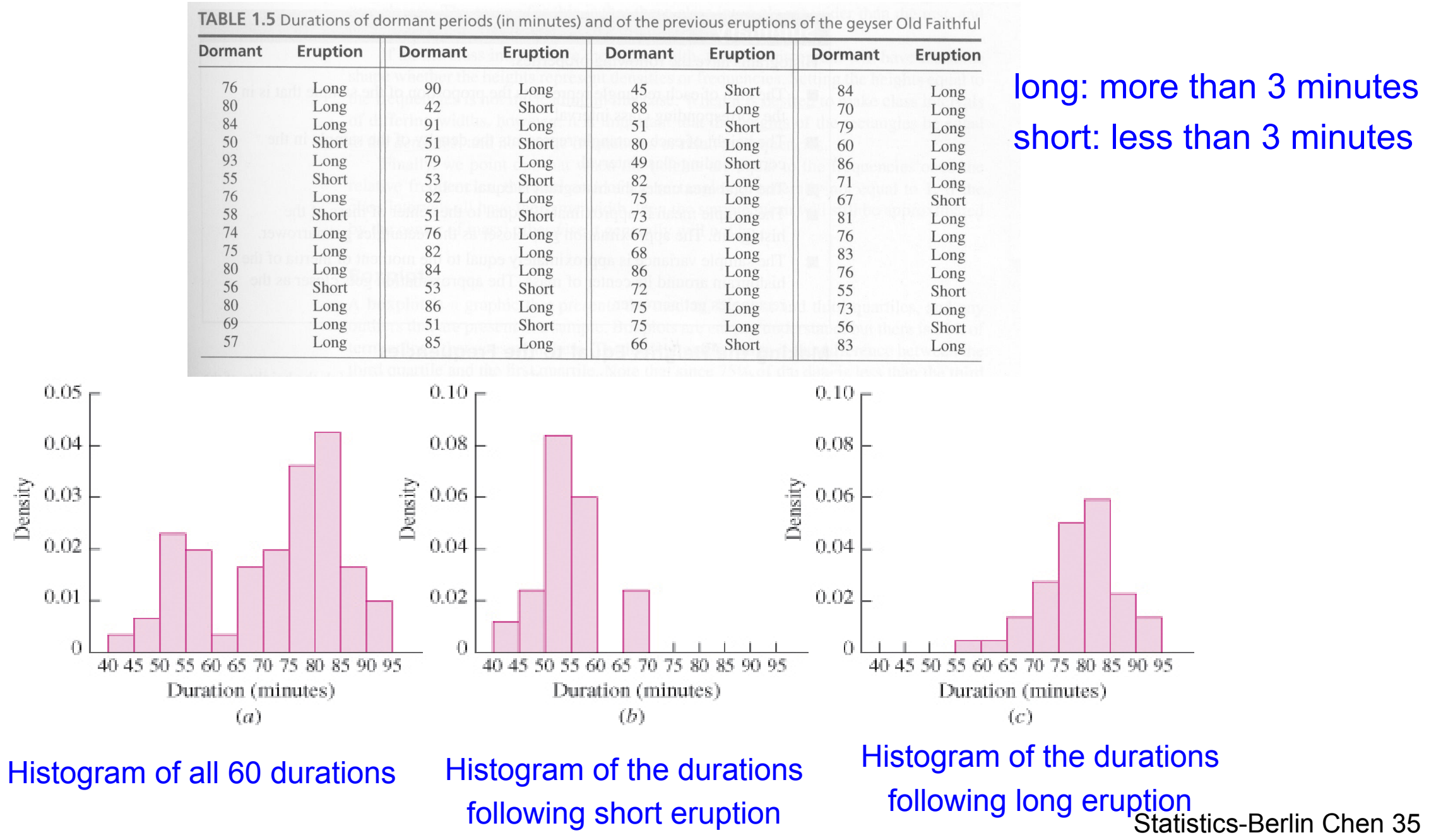




\section{Histogram with Height Equal to Frequency}

- Till now, we refer the term "histogram" to a graph in which the heights of rectangles represent densities

- However, some people draw histograms with the heights of rectangles equal to the frequencies

- Example: The histogram of the sample in Table 1.4 with the heights equal to the frequencies
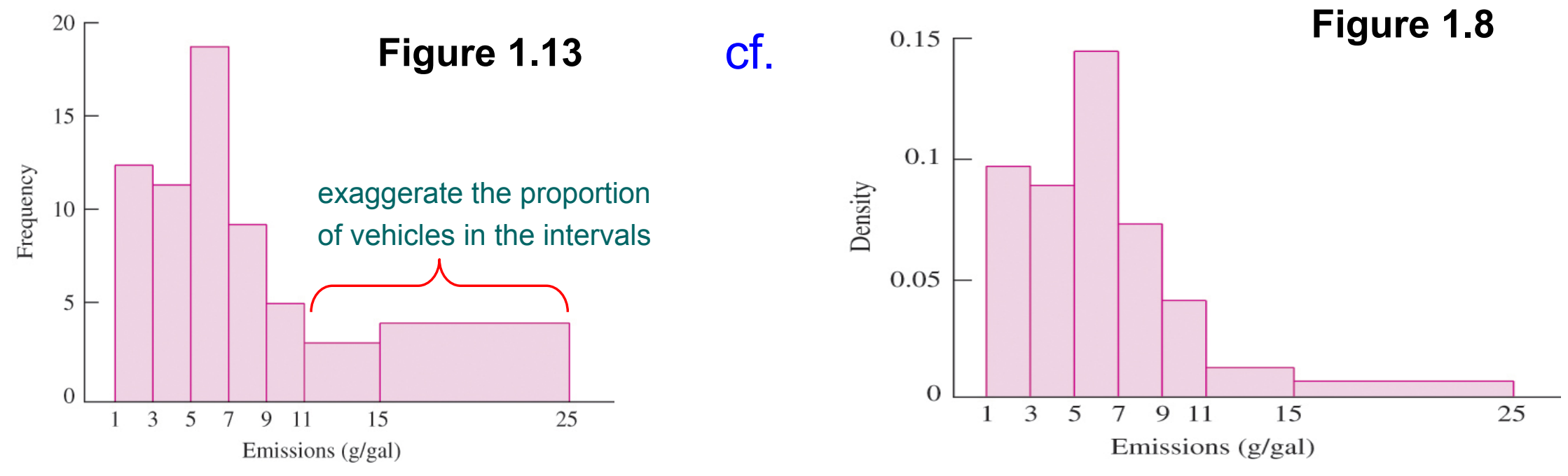


\section{Boxplot (1/4)}

- A boxplot is a graph that presents the median, the first and third quartiles, and any outliers present in the sample

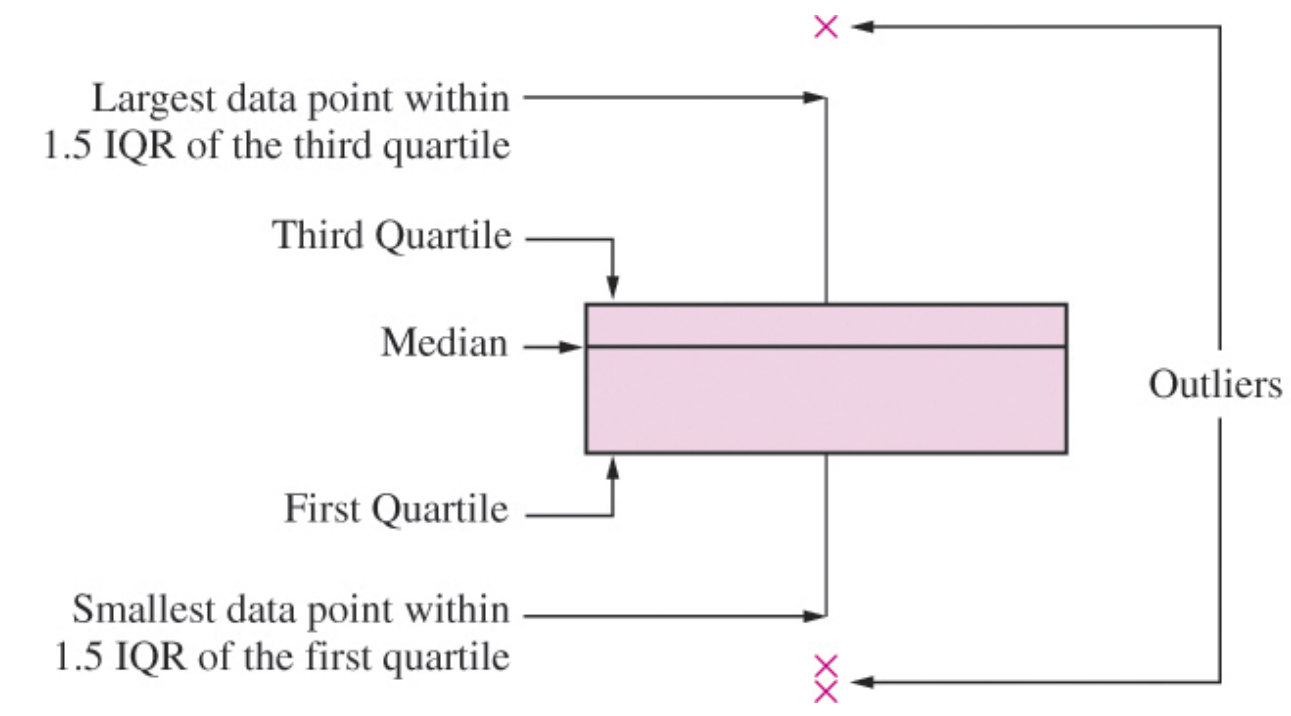

- The interquartile range (IQR) is the difference between the third and first quartile. This is the distance needed to span the middle half of the data 


\section{Boxplot (2/4)}

- Steps in the Construction of a Boxplot

- Compute the median and the first and third quartiles of the sample. Indicate these with horizontal lines. Draw vertical lines to complete the box

- Find the largest sample value that is no more than 1.5 IQR above the third quartile, and the smallest sample value that is not more than 1.5 IQR below the first quartile. Extend vertical lines (whiskers) from the quartile lines to these points

- Points more than 1.5 IQR above the third quartile, or more than 1.5 IQR below the first quartile are designated as outliers. Plot each outlier individually 


\section{Boxplot (3/4)}

- Example: A boxplot for the geyser data presented in Table 1.5

- Notice there are no outliers in these data

- The sample values are comparatively densely packed between the median and the third quartile

- The lower whisker is a bit longer than the upper one, indicating that the data has a slightly longer lower

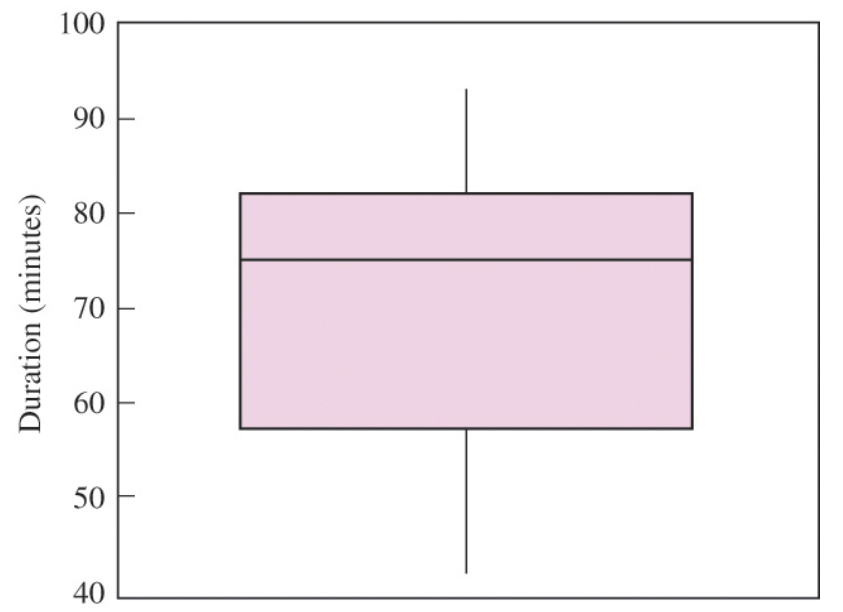
tail than an upper tail

- The distance between the first quartile and the median is greater than the distance between the median and the third quartile

- This boxplot suggests that the data are skewed to the left 


\section{Boxplot (4/4)}

- Another Example: Comparative boxplots for PM emissions data for vehicle driving at high versus low altitudes

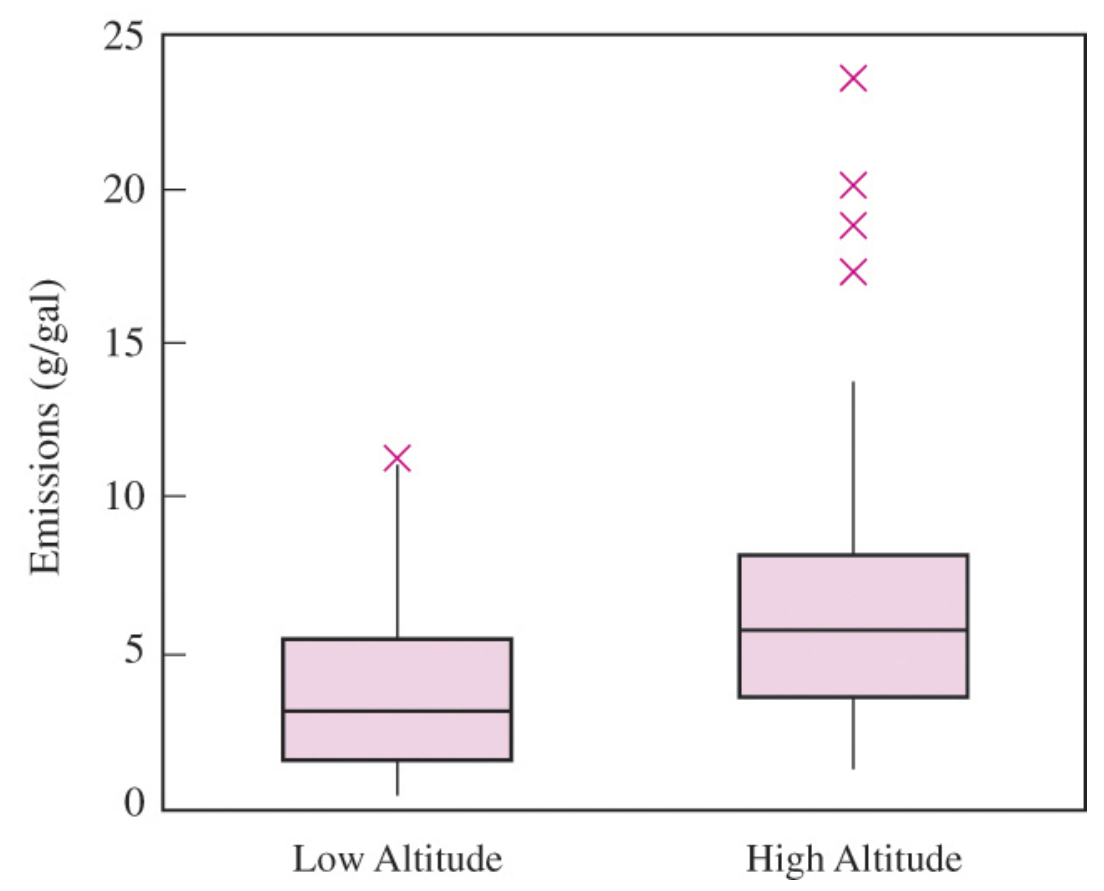




\section{Scatterplot (1/2)}

- Data for which item consists of a pair of values is called bivariate

- The graphical summary for bivariate data is a scatterplot

- Display of a scatterplot
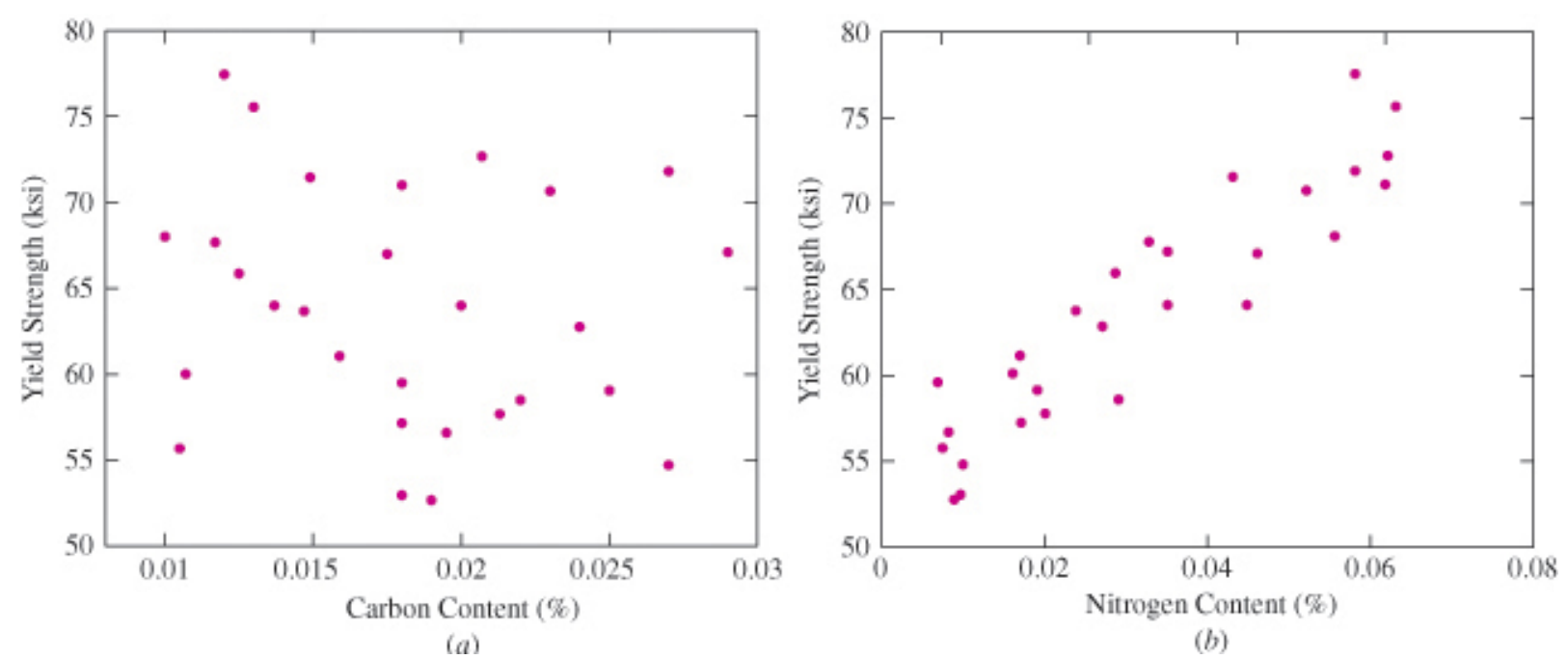


\section{Scatterplot $(2 / 2)$}

- Example: Speech feature sample (Dimensions $1 \& 2$ ) of male (blue) and female (red) speakers after LDA transformation

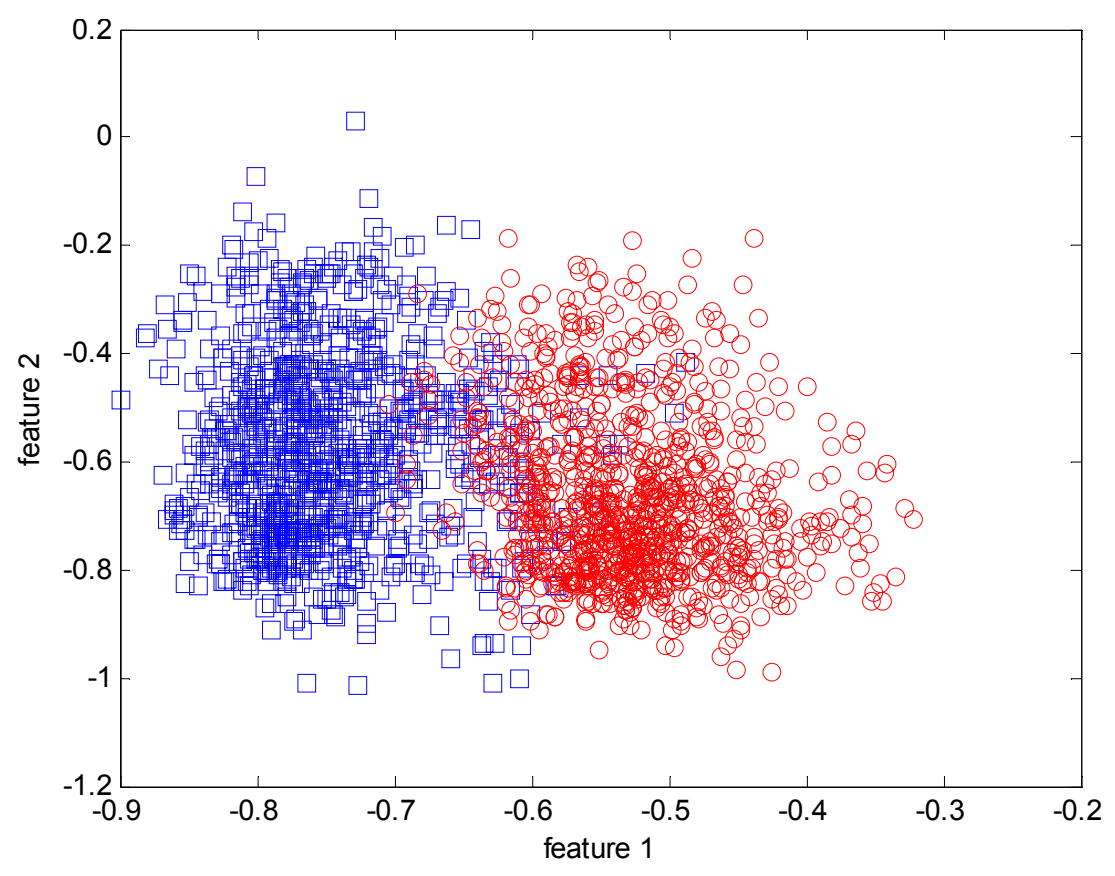




\section{Summary}

- We discussed types of data

- We looked at sampling, mostly SRS

- We learned about sample statistics

- We examined graphical displays of data 